\title{
Nutritional Programming of Probiotics to Promote Health and Well-Being
}

\author{
Alice Maayan Elad and Uri Lesmes \\ Additional information is available at the end of the chapter
}

http://dx.doi.org/10.5772/50051

\section{Introduction}

The human large intesine is inhabited by a diverse and complex bacterial flora, which includes an outstanding total number of $10^{14}$ cells, $>1000$ species and a biomass of more than $1 \mathrm{~kg}[1,2]$. Thus, the gut microbiota may be conceived as a specialized 'microbial organ' within the gut, affecting human health and disease through its involvement in pathogenesis, nutrition and immunity of the host [1-3]. Recently it has also been recognized that this dynamic yet stable ecosystem plays a role in conditions such as obesity and diabetes as well as in general well-being, from infancy to ageing [1-8]. Consequently, an increasing number of studies which explore the potential of promoting health by nutrition focuses on possible ways to influence and modulate the composition and activity of the gut flora towards a healthier one $[4,9-12]$.

In this respect, three major dietary approaches have been studied and applied. The first approach of probiotics is to fortify the gut flora through the consumption of exogenous live microorganisms, e.g. L. acidophilus in dairy products. The second strategy of prebiotics seeks to selectively stimulate the growth and/or activity of one or a limited number of advantageous indigenous bacteria in the host gut flora [1,13]. The third approach, known as synbiotics due to its synergistic nature, aims to combine the previous ones by the simultaneous administration of probiotics and prebiotics, which improves the survival and implantation of the live microbes [13].

Over the years, much attention has been drawn to indigestible carbohydrates that evade enzymatic digestion in the upper gastrointestinal tract and become available for fermentation in the colon [13]. These dietary compounds were later termed as prebiotics, a definition of which has been updated into its current form as "a selectively fermented ingredient that allows specific changes, both in the composition and/or activity in the gastrointestinal microflora that confers benefits upon host well-being and health" [14, 15]. 
Although a more recent development compared to probiotics, prebiotics have been at the heart of various studies and numerous commercial products since they do not share the problem of probiotic survival upon ingestion by the consumer, and they can be added to a broad range of food products (e.g. confectionary and baked foods as well as more traditional fermented milk products and fruit drinks) because the majority of prebiotics are carbohydrates [16].

Amongst the carbohydrates currently marketed as prebiotics, inulin, fructo-oligosaccharides (FOS), galacto-oligosaccharides (GOS) and lactulose are consistently supported by high quality data from in vitro, in vivo and human trials [10-12, 14, 16-19]. Specifically, human trials have established that dietary consumption of 5-20 g/day of these prebiotics stimulates the growth of Bifidobacterium and Lactobacillus and promotes the health and well-being of infants, adults, pregnant and lactating women as well as the elderly to varying extents $[6,8$, $11,20,21]$.

\section{Prebiotics as gut flora management tools}

\subsection{Established prebiotics}

Overall, three major groups of compounds have been consistently established as prebiotics conferring health benefits (as detailed in Table 1): fructans, which include inulin and fructooligosaccharides (FOS), galacto-oligosaccharides and lactulose. Under the general term fructans one can classify three established prebiotic carbohydrates: inulin, fructooligosaccharides (FOS) and short chain fructo-oligosaccharides (scFOS) [16-18]. The fructans are polymers composed of $\mathrm{D}$-fructose units joined by $\beta-2-1$ glycosidic linkages and terminated by an $\alpha-1-2$-linked D-glucose.

\begin{tabular}{|c|c|c|c|c|}
\hline $\begin{array}{l}\text { Established } \\
\text { prebiotic }\end{array}$ & $\begin{array}{l}\text { Recommended } \\
\text { efficaceous } \\
\text { intake }[g / \text { day] }\end{array}$ & $\begin{array}{l}\text { Key effects in } \\
\text { humans }\end{array}$ & $\begin{array}{c}\text { Potential } \\
\text { adverse intake } \\
\text { [g/day] }\end{array}$ & $\begin{array}{l}\text { Suggested } \\
\text { references }\end{array}$ \\
\hline Inulin & $5-15$ & $\begin{array}{ll}\text { - } & \text { Stimulate } \\
\text { bifidobacteria }\end{array}$ & $\begin{array}{l}>15 \text { (increase in } \\
\text { fecal output) }\end{array}$ & [22] \\
\hline $\begin{array}{l}\text { Fructo- } \\
\text { oligosaccharides }\end{array}$ & $10-15$ & $\begin{array}{l}\text { growth } \\
\text { Production of }\end{array}$ & $\begin{array}{l}>15 \text { (increase in } \\
\text { fecal output) }\end{array}$ & [17] \\
\hline $\begin{array}{l}\text { Galacto- } \\
\text { oligosaccharides }\end{array}$ & $10-15$ & $\begin{array}{l}\text { short chain fatty } \\
\text { acids }\end{array}$ & $\begin{array}{l}>20 \mathrm{~g} / \text { human } \\
\text { body (diarrhea) }\end{array}$ & [19] \\
\hline Lactulose & 10 & $\begin{array}{l}\text { Protection } \\
\text { against enteric } \\
\text { infections }\end{array}$ & $>20$ (laxative) & [23] \\
\hline
\end{tabular}

Table 1. The main established prebiotics and their beneficial/adverse intakes.

The degree of polymerization (DP), defined by the number of monosaccharide units, is used to distinguish between inulin, FOS and scFOS. Molecules with a DP between 2-60 are referred to as inulin. Inulin is commercially produced from chicory roots, but it is present in 
varying extent also in onions, garlic, Jerusalem artichoke, tomato and banana [11, 16, 21]. Similarly, oligofructose, commonly referred to as FOS, is prepared from chicory in an enzymatic hydrolysis using inulinase, and defined as oligosaccharide fractions which have a maximal DP of 20 with most common commercial products having an average DP of 9. In contrast, scFOS are synthesized in an enzymatic reaction via transfer of fructosyl units from sucrose molecules to yield mixtures of fructosyl chains with a maximum DP of 5 . The mixture produced is usually comprised mainly of 1-kestose ( 2 units of fructose linked to glucose, GF2), nystose (GF3) and 1-fructosyl nystose (GF4) [16, 17, 24].

Fructans have a long tradition as prebiotics. Since their fructose units are joined by $\beta$ linkages, they are resistant to hydrolysis by the human digestive enzymes which mainly cleave $\alpha$-linkages. As a consequence, when these carbohydrates reach the colon they selectively stimulate the growth of beneficial bacteria such as bifidobacteria, which do contain specific enzymes for their degradation, i.e. $\beta$-fructosidases $[16,21,25]$. Therefore, inulin, FOS and scFOS are classified as 'nondigestible' carbohydrates, with a calorie value of $1.5-2.0 \mathrm{kcal} / \mathrm{g}$ [24]. FOS fermentation in the colon results in increased levels of short chain fatty acids (SCFA) which lower the $\mathrm{pH}$ in the intestinal lumen. This can provide an explanation to the reports that these fructans lead to a decrease in the number of harmful bacteria in the colon (such as Clostridium, Streptococcus faecallis and Escherichia coli) [21, 25].

Galacto-oligosaccharides (GOS) are galactose-containing oligosaccharide mixtures of the form Glu $\alpha-1-4[\beta-$ Gal-1-6]n where $n$ can be between two to five. They are produced from lactose syrup using $\beta$-galactosidases, which catalyze the hydrolysis of lactose into glucose and galactose, and also the transgalactosylation reactions with lactose as acceptor of galactose units giving rise to a variety of glycosidic linkages and molecular weights [11, 16, $19,21]$. Furthermore, the use of different enzymes in the various production processes of GOS leads to variability in their purity and glycosidic linkages, with $\beta-1-6, \beta-1-3$ and $\beta-1-4$ being the dominant [19]. Several in vitro and in vivo experiments have demonstrated that as in inulin-type fructans, the $\beta$-glycosidic linkages in GOS render them resistant to hydrolysis by the human digestive enzymes secreted in the upper gastrointestinal tract $[16,19,21,26]$. In light of that, manufacturers are obliged by the European regulation to clearly identify GOS-containing food products as dietary fibers, with an estimated low calorie value of 1-2 $\mathrm{kcal} / \mathrm{g}$ [19].

Most of the health effects related to GOS arise from their selective fermentation by bifidobacteria and lactobacilli. In fact, it has been reported that when added to infant milk formulas, these oligosaccharides replicated the bifidogenic effect of human breast milk, not only in bacterial counts, but also with respect to the metabolic activity of the microflora in the colon [16, 27]. The growth of Lactobacillus paracasei and Bifidobacterium lactis has been shown to be preferential when grown on tri- and tetrasaccharide fractions of FOS or GOS, which supports the notion that prebiotics selectively promote the proliferation of bacteria possesing an active transport system enabling them to utilize these oligosaccharides [28-30]. In addition, it has been demonstrated that GOS compete for pathogen binding sites that coat the surface of the gastrointestinal epithelial cells [31, 32]. 
Finally, lactulose ( $\beta$-1-4-galactosyl-fructose) is a synthetic disaccharide derived from lactose. It is commonly used as a laxative in pharmaceutical products for the treatment of constipation, in doses over $20 \mathrm{~g} /$ day $[16,21]$. Nevertheless, human trials have shown that at lower doses, lactulose acts as a prebiotic, reaching the colon and increasing bifidobacteria counts $[2,16,17,23]$. Although this substance is an established prebiotic, it is still heavily confined to applications as a therapeutic agent.

\subsection{Novel prebiotics}

The search for new and novel prebiotics is constantly driven by the increased interest in management of human health through nutrition, particularly by the modulation of gut flora. In addition, studies of established prebiotics have enabled the better understanding of mechanisms of action and properties, which provided the basis for emerging prebiotics. Among the large array of prebiotic candidates, isomalto-oligosaccharides (IMO), xylooligosaccharides (XOS), soy-oligosaccharides (SOS), gluco-oligosaccharides, lactosucrose and resistant starches can be classified as emerging prebiotics [17, 33-40]. Some of these compounds present advantages over established prebiotics; for example, XOS are stable across a wide range of $\mathrm{pH}$, hence they are resistant to degradation in low $\mathrm{pH}$ juices, in contrast to inulin [21]. Besides attempts to identify and isolate naturally occurring prebiotics there are also attempts to enhance and extend the functionality of exisiting natural prebiotics through a rational design approach [41].

Promising results have been reported, including the selective growth of bifidobacteria and lactobacilli and/or the formation of beneficial metabolites, such as short chain fatty acids. However, it should be noted that these studies are still limited to in vitro models or small scale animal or human trials [16, 17, 40]. For example, thermally produced resistant starch has been demonstrated to possess a bifidogenic and butyrogenic effect in an in vitro three stage continuous fermentation system inoculated with human feces [42]. Furthermore, this study suggested that resistant starch crystalline polymorphism, resulting from different thermal treatments, could convey different prebiotic effects on the human colon flora.

\section{Efficacy of prebiotics across the life span}

\subsection{Methods to evaluate prebiotics}

Research into the efficacy of prebiotics includes a collection of methods currently in use, from pure cultures to human trials, which can be generally classified into in vivo and in vitro methods. Overall, the prebiotic effect is mainly evaluated by the presence of beneficial metabolites and measuring the growth of major bacterial groups commonly present in the human gut, in particular a selection for increased numbers of bifidobacteria and lactobacilli in comparison with undesirable bacteria such as certain clostridia and sulfate reducing bacteria [40]. Ultimately, health claims concerning prebiotic effects must rely on comprehensive well-controlled human trials. Thus, in vivo studies have evolved over the years to robust experimental designs which combine double blind and placebo-controlled 
designs with advanced microbial analyses, such as bacterial enumeration using 16s DNA probes in fluorescence in situ hybridization (FISH) [16]. In most human studies, the production of short chain fatty acids (SCFA) has been quantified in fecal samples, as a marker of enhanced saccharolytic fermentation in response to prebiotic treatment [13].

In spite of their high significance, in vivo human studies are usually limited, mainly due to financial and ethical restrictions. Therefore, animal models have been used as a possible viable alternative to the human GI tract, while allowing the researchers to perform in vivo experiments in tightly controlled conditions as well as access intestinal contents, tissues and organs at autopsy. Moreover, many in vivo experiments have used germ-free animals dosed with fecal suspensions obtained from human donors, which are considered to be a reliable model for a reconstituted human gut flora. However, data generated from animal models do not necessarily coincide with human or in vitro studies, as has been shown for prebiotic resistant starch type III [42].

Consequently, many in vitro experimental models have been developed to simulate various aspects of the human GI tract [43-45]. Seeking to closely mimic the conditions of organs along the GI tract, these models include a reactor or a series of reactors under tightly controlled settings, with the large intestine represented by an anaerobic reactor/s inoculated with fecal slurries [40]. Thus, these systems offer researchers a controlled experimental design that is relatively inexpensive, easy to set up, high throughput and raises minimal ethical issues [46].

One of the first in vitro GI models described in the literature was termed the simulator of the human intestinal microbial ecosystem (SHIME) [47]. This computer controlled model is composed of a five serially connected vessels simulating the conditions of the stomach, small intestine, ascending, transverse and descending colon [48, 49]. Operators can control various parameters of physiological relevance, including gastric and pancreatic secretions, $\mathrm{pH}$, transit time, feed composition as well as sample different loci along the system on a regular basis [13, 46]. Another comprehensive in vitro GI model was developed in the Netherlands [50]. This model is actually comprised of two seperate parts: TIM-1 is a series of four computer controlled chambers simulating the upper GI, i.e. the stomach, duodenum, jejunum and ileum, while TIM-2 models the large intestine. Unlike the SHIME, this model consists of a series of linked glass vessels containing flexible walls, which allow simulation of the peristaltic movements of the GI. The hollow fiber membrane construct of the system enables to simulate absorption of water and nutrients in the lumen as well as their removal from the colon $[13,46]$. Similarly, simple glass reactors have been used for in batch and three stage continuous fermentation systems to simulate the lower GI, i.e. the proximal, transverse and distal colon, and have been validated against sudden death victims [51].

Overall, in vivo methods and particularly human trials are essential for establishing health claims regarding prebiotic effects on human microflora. However, such methods are hindered by financial, ethical and practical reasons. In vitro models fail to fully mimic the GI system, particularly peristaltic movements, mucosal uptake and impact of immune components. Nevertheless, these systems offer relatively low costs, ease of use and have 
minimal ethical considerations, while providing researchers controllable settings for studying luminal biochemistry and microbiology.

\subsection{Prebiotic efficacy in infancy and childhood}

At birth, the neonate gut is considered to be sterile with rapid colonization by bacteria believed to occur in three phases: delivery, breastfeeding and weaning to solid foods [52-54]. Immediately after birth, facultative anaerobic bacteria such as Enterobacteriaceae, streptococci and staphylococci colonize the gut environment of the newborn, gradually consuming oxygen and producing various metabolites. Consequently, strict anaerobic bacterial population dominated by bifidobacteria, Clostridium and Bacteroides can be established [5557]. Within the first year of life, the microflora is highly dynamic, but by the age of two years with the introduction of solid foods, the colonic microbiota is considered complete - it stabilizes and resembles that of the adult [58-60].

The interest in prebiotics as a nutritional strategy to program infant gut microbiota to favor a more advantageous population has been inspired by the beneficial effects attributed to the 200 different human milk oligosaccharides (HMO) [52-54]. Based on the observations of bifidobacteria in the feces of breastfed babies, attempts have been made to reproduce this bifidogenic aspect in infant formulas by adding commercial prebiotics, in particular FOS and GOS $[8,53]$. This practical application of prebiotics was evaluated for example in double-blind, randomized and controlled studies in 90 full term infants, which demonstrated that $4 \mathrm{~g} / \mathrm{L}$ or $8 \mathrm{~g} / \mathrm{L}$ of FOS, GOS or their combination resulted in a significant decrease in fecal $\mathrm{pH}$ and a concomitant increase in bifidobacteria and lactobacilli after 28 days feeding [61, 62]. A GOS and FOS mixture at a ratio of 9:1 (GOS:FOS) has been extensively studied as a prebiotic additive to infant formulas [63], and shown to increase bifidobacteria in infant feces and lower the incidence of pathogens [64-66]. Therefore, the administration of FOS and GOS into commercial infant formulas for their prebiotic effects has spread, and researchers continue exploring additional prebiotics as possible candidates for infant formulas supplementation. Moreover, studies are also looking into the persistence of the prebiotic effects.

\subsection{Prebiotic efficacy in adulthood}

To date, various studies have determined fructans (inulin and FOS) induce different beneficial effects on the health and general well-being of healthy adult subjects [10, 16-18]. A daily consumption of 5-10 $\mathrm{g}$ of fructans has been demonstrated to exert a bifidogenic effect on healthy adults based on dose-response studies, while similar doses of GOS and lactulose have been reported in in vitro and human trials as stimulating a bifidogenic effect $[16,67$, 68]. As to bowel habit, i.e. the frequency of bowel discharge but not fecal output, constipation and laxative effect, there is some evidence that in constipated subjects, inulin may increase bowel habit [69], whereas lactulose is prescribed at $20 \mathrm{~g} /$ day to increase fecal output of chronically constipated patients, however, having a bifidogenic effect on healthy adults at lower doses [68]. Moreover, numerous studies have shown that fecal output 
remains unchanged at a daily intake of up to $15 \mathrm{~g}$ fructans with a slight increase at doses of $15 \mathrm{~g} /$ day or higher [11]. Hence, inulin, FOS, GOS and lactulose may be defined as mild laxatives with adverse effects observed only at a consumption of over $20 \mathrm{~g} / \mathrm{day}$.

Additionally, established prebiotics have been linked to protection against enteric infections, modification of the host immune response, production of short chain fatty acids, particularly butyrate, increased mineral absorption and even the reduced risk of colon cancer [10, 11, 14, $16,18]$. Prebiotics efficacy has also been studied during pregnancy and lactation. These life periods are sometimes accompanied by irregular gastrointestinal activity, which can be improved by the consumption of dietary fibers and prebiotics [20]. Furthermore, gestational weight gain and postpartum weight retention have been suggested to be affected from prebiotics intake, since they modulate the gut microflora [5]. However, it is also important to note that the prebiotic effect has not been found to extend to neonates and infants, even when solely breast fed.

\subsection{Prebiotics efficacy in ageing}

At the old age, increased threshold for taste and smell as well as masticatory dysfunction can lead to a nutritionally imbalanced diet. In addition, various physiological functions deteriorate with age and may influence the absorption and/or metabolism of nutrients. Furthermore, the increased intake of drugs results in GI disturbances due to antibiotics undesired effect on indigenous bacteria in the host gut flora. Thus, changes in the GI tract, modification of diet and host immune system inevitably give rise to bacterial population alterations [70, 71]. In spite of the increasing proportion of the elderly in Western countries $[72,73]$, scarce data exists on the changes that occur in the intestinal microbiota during the ageing process and their possible health outcomes. Overall, an increase in facultative anaerobes and decrease in Bacteroides and bifidobacteria (total numbers as well as species diversity) have been reported [74].

Therefore, modulation of the colon microflora by the consumption of prebiotics is increasingly being studied as a potent, cost effective and natural way to improve the health and well-being of elderly people as well as reduce risks for various diseases [70, 71, 74]. FOS and GOS ingestion, as well as synbiotic preparations, were found to significantly increase the number of bifidobacteria at the expense of less beneficial microbiota in ageing individuals [6, 75-78]. In addition, a randomized, double-blind, controlled study with 74 subjects aged 70 and over has indicated that prebiotic addition can improve the low noise inflammatory process frequently observed in this sensitive population [79].

\section{Prebiotics as therapeutics}

\subsection{Prebiotics therapeutic efficacy in human diseases}

It is now well documented that the bacteria microflora residing in the human GI has a role not only in promoting health but also in preventing some diseases [3, 80, 81]. Prebiotics 
have been reported to protect against pathogenic gastrointestinal infections by promoting the growth of probiotics which help displace pathogens from the mucosa, producing antimicrobial agents and competing with pathogens on binding sites and nutrients [3]. In addition to in vitro data which supports this disease preventing effect of prebiotics [16, 22], a human study on 140 infants has concluded that consumption of oligofructose and cereal significantly reduced events of fever, frequency of vomiting, regurgitation and abdominal discomfort [82]. Moreover, various studies have shown that prebiotics can beneficially affect patients with antibiotic-associated diarrhea, especially when it arises from C. difficile [11].

Prebiotics have also been reported to reduce the risk of colon cancer as a result of gut flora modulation [11, 14, 16-18]. Specifically, they support the metabolism of carcinogenic molecules and the secretion of short chain fatty acids to the lumen by the colon microbiota $[83,84]$. Furthermore, human trials have demonstrated that inulin, FOS and scFOS beneficially affect colorectal cell proliferation and genotoxicity [17], hence the potential of prebiotics in prevention and treatment of colon cancer should be further explored.

Inflammatory bowel disease (IBD), which includes ulcerative colitis (UC) and Crohn's disease (CD), has also been researched as a possible target for prebiotics [11, 16, 17]. As mucosal communities significantly change in these diseases, prebiotics may be used in order to manipulate them. For example, patients fed $15 \mathrm{~g}$ per day of a prebiotic mixture composed of $7.5 \mathrm{~g}$ inulin and $7.5 \mathrm{~g}$ FOS for 2 weeks prior to colonoscopy, have had more than a 10-fold increase in bifidobacterial and eubacterial numbers in the mucosa of the proximal and distal colon [85]. Similarly, in a small open-label human trial, 10 patients with active ileum-colonic CD were fed $15 \mathrm{~g}$ FOS daily for 3 weeks, after which a significant reduction in the Harvey Bradshaw index of disease activity was observed as well as an increase of fecal bifidobacteria numbers [11].

\subsection{Prospective therapeutic targets}

\subsubsection{Obesity and the metabolic syndrome}

Increasing evidence linking gut flora to human health and diseases have inspired further research regarding the possible link between gut flora and obesity, which has led to the notion that prebiotics could be harnessed as potential therapeutic agents or management tools to prevent and treat obesity and the metabolic syndrome [4, 5, 7, 20,52]. The metabolic syndrome is a cluster of metabolic abnormalities, including abdominal obesity, type 2 diabetes mellitus and cardiovascular diseases [86, 87].

Various studies have shown that prebiotic intervention decreased fat storage in white adipose tissues and in the liver, decreased hepatic insulin resistance as well as systemic inflammation in several nutritional (high-fat diet-fed) and genetic (ob/ob mice) obese rodents [88-95]. Some beneficial effects of fructans on BMI, fat mass and insulin resistance were also shown in the limited human trials conducted so far [95-98]. 
However, to date, the mechanisms underlying the complex role of gut microbiota in such conditions are largely unknown [1]. It has been reported that a lower number of bifidobacteria at birth is associated with overweight later in childhood [99], and in adults, the number of bifidobacteria is slightly lower in individuals with obesity than in lean subjects [100]. The number of these bacteria is also decreased in patients with type 2 diabetes mellitus compared with nondiabetic people [101]. Hence, these results seem to suggest that bifidobacteria affects the development of obesity and its related comorbidities [52].

A remarkable increase has been observed in the number of Bifidobacterium spp. following inulin-type fructans supplementation to mice with diet-induced or genetically determined obesity [52]. Interestingly, the number of bifidobacteria was inversely correlated with the development of fat mass, glucose intolerance and levels of lipopolysaccharides (LPS) [102]. LPS has been found at a significantly higher level in the serum of obese individuals, which creates a metabolic endotoxemia, leading to obesity, insulin resistance and systemic inflammation [103]. Moreover, it has been reported that the overexpression of numerous host genes that are related to adiposity and inflammation was prevented by prebiotic intake [52].

A pathway involving short chain fatty acids (SCFA) has been proposed to be involved in the interplay between prebiotics, the gut flora and obesity. SCFA act as signaling molecules and are specific ligands for at least two G protein-coupled receptors, GPR41 and GPR43, which have a potential role in fat mass development [13, 104]. In addition, it has been shown that acetate and propionate can modify hepatic lipid metabolism [105]. Interestingly, a recent study has demonstrated that diet-induced obesity and insulin resistance were prevented when mice on a high-fat diet were supplemented with butyrate, which promoted energy expenditure and induced mitochondrial function [106]. Various studies have shown that a diet enriched with prebiotics leads to a greater intestinal SCFA production and thereby migitates body weight gain, fat mass development and the severity of diabetes $[89,90,100$, 107-109]. Numerous peptides secreted by the enteroendocrine cells along the GI are involved in the regulation of energy homeostasis and/or pancreatic function. Three such peptides which can modulate food intake and energy expenditure are glucagon-like peptide-1 (GLP-1), peptide YY (PYY) and ghrelin [110-113]. Thus, it has been suggested that SCFA are related to changes in the gut peptide secretion, namely increased production and secretion of GLP-1 and PYY and the reduction of ghrelin which induce metabolic effects [13, 114, 115]. Piche et al. were the first to report that inulin-type fructan feeding of $20 \mathrm{~g} / \mathrm{day}$ significantly increased plasma GLP-1 in humans [116]. In another study, a 2-week supplementation with inulin-type fructans (16 g/day) to healthy volunteers increased GLP-1, consequently increasing satiety, lowering calorie intake and decreasing postprandial glycemia [90, 108]. Furthermore, prebiotic treatment in obese patients has been found to induce and increase PYY and decrease ghrelin levels [117]. Finally, Tarini and Wolever have demonstrated that a single dose of inulin significantly increased postprandial plasma GLP-1 and decreased plasma ghrelin [118]. This is in contrast to perceived necessity for a prolonged prebiotics administration to modulate gut microbiota and allow effect on gut endocrine function. Thus, it seems that further studies are needed to fully unravel the 
potential of prebiotics to offer a nutritional means to cope with the worrisome increase in human obesity and the metabolic syndrome.

\section{Future challenges}

\subsection{Harmonization of methods to evaluate efficacy}

To date, a plethora of studies have investigated the effects of prebiotics on human health and well-being, leading to the general realization prebiotics could serve as a possible method of therapeutic intervention. However, the majority of these studies cannot be compared due to the variety of methods employed. For example, results obtained by bacterial isolation techniques $[39,119]$ cannot be compared with data from more advanced methods for the molecular characterization of the microbiota, now considered essential to obtain a comprehensive view of the gut ecosystem. Furthermore, DNA-based techniques including the use of the 16s ribosomal RNA gene are considered as less biased, hence their results are more reliable [54]. In addition, studies focused on the effect of prebiotics on the elderly, even in healthy subjects, lack a clear definition of 'elderly', and various groups are recruited, usually on the basis of 'over 60' [120], 'over 65' [119, 121] or 'over 70' [122]. This makes is difficult to define a 'threshold age' at which the gut environment starts to be influenced from the ageing process [70]. Thus, harmonization of methods to evaluate efficacy is a prequisite step limiting the further application of prebiotics for the prevention and treatment of diseases as well as the development of novel prebiotics.

\subsection{The challenge of personalization}

A broad range of parameters has been known to affect the bacterial composition of the infant gut, e.g. mode of delivery, type of feeding (exclusive breastfeeding versus formula), antibiotic use and maternal infection [55-57, 123, 124]. Furthermore, various studies have indicated that the low number of certain bacteria at birth such as bifidobacteria is related to overweight later in childhood [99]. Consequently, prebiotics supplementation even in infants may be used as a preventive nutritional programming tool, which will affect the health and well-being also in adulthood. Moreover, it has been increasingly accepted that environmental factors such as nutritional habits and lifestyle may impact the gut microbiota composition, for example striking country-related differences in the effects of age on the microflora have been reported [70]. This wide variety of factors, affecting intestinal microbiota composition from infancy to elderly, drove the need for personalized nutrition, including personalized and tailored prebiotics. In addition, since prebiotics are recently considered even as therapeutic agents for the prevention and treatment of diseases, it may be beneficial to aim personalized prebiotics to people at high-risk to develop these illnesses. Thus, the challenge of personalization includes performing long-term, large-sized, wellcontrolled and multidisciplinary-collaborated studies which will demonstrate and establish the health promoting effects of prebiotics and enable harnessing them in the clinic or in supermarket shelves. 


\section{Conclusions}

Prebiotics have emerged as cost-effective and efficient nutritional programming tools to beneficially and selectively promote the growth and/or activity of certain bacteria in the indigenous flora of the human GI. So far, prebiotics have been demonstrated to exert various beneficial effects during an individual's lifetime, from infancy to ageing, as well as function as therapeutic agents for the prevention and treatment of different diseases, including obesity and the metabolic syndrome.

In addition to the well-established prebiotics of FOS, GOS and lactulose, novel prebiotics are constantly being developed. State of the art techniques, in vitro gastrointestinal models and advanced computerization tools are leading many researchers to adopt more complete and comprehensive approaches, e.g. metabolomics and metagenomics.

Future challenges include the harmonization of methods of evaluating efficacy that will help focus research efforts and enable a more comprehensive understanding of prebiotic mechanisms of action and beneficial effects and how these can be modulated. Another important prospect is personalization, i.e. fitting tailored prebiotics to individual needs in order to nutritionally program and affect their health and well-being from infancy and into old and prosperous age.

\section{Author details}

Alice Maayan Elad and Uri Lesmes

Department of Biotechnology and Food Engineering, Technion, Israel Institute of Technology, Haifa, Israel

\section{References}

[1] Diamant, M., E.E. Blaak, and W.M. de Vos, Do Nutrient-Gut-Microbiota Interactions Play a Role in Human Obesity, Insulin Resistance and Type 2 Diabetes? Obesity Reviews, 2011. 12(4): p. 272-281.

[2] Venema, K., Intestinal Fermentation of Lactose and Prebiotic Lactose Derivatives, including Human Milk Oligosaccharides. International Dairy Journal, 2012. 22(2): p. 123-140.

[3] O'Hara, A.M. and F. Shanahan, The Gut Flora as a Forgotten Organ. EMBO Rep, 2006. 7(7): p. 688-693.

[4] Delzenne, N.M. and P.D. Cani, Nutritional Modulation of Gut Microbiota in the Context of Obesity and Insulin Resistance: Potential Interest of Prebiotics. International Dairy Journal, 2010. 20(4): p. 277-280.

[5] Duncan, S.H., et al., Human Colonic Microbiota Associated with Diet, Obesity and Weight Loss. International Journal of Obesity, 2008. 32(11): p. 1720-1724.

[6] Tuohy, K.M., Inulin-Type Fructans in Healthy Aging. The Journal of Nutrition, 2007. 137(11): p. 2590S-2593S. 
[7] Tuohy, K.M., A. Costabile, and F. Fava, The Gut Microbiota in Obesity and Metabolic Disease - A Novel Therapeutic Target. Nutritional Therapy and Metabolism, 2009. 27(3): p. 113-133.

[8] Parracho, H., A.L. McCartney, and G.R. Gibson, Probiotics and Prebiotics in Infant Nutrition. Proceedings of the Nutrition Society, 2007. 66: p. 405-411.

[9] Fooks, L.J., R. Fuller, and G.R. Gibson, Prebiotics, Probiotics and Human Gut Microbiology. International Dairy Journal, 1999. 9(1): p. 53-61.

[10] Gibson, G.R., Prebiotics as Gut Microflora Management Tools. Journal of Clinical Gastroenterology, 2008. 42(6): p. S75-S79.

[11] Macfarlane, S., G.T. Macfarlane, and J.H. Cummings, Review Article: Prebiotics in the Gastrointestinal Tract. Alimentary Pharmacology \& Therapeutics, 2006. 24(5): p. 701-714.

[12] Tuohy, K.M., et al., Using Probiotics and Prebiotics to Improve Gut Health. Drug Discovery Today, 2003. 8(15): p. 692-700.

[13] De Preter, V., et al., The Impact of Pre- and/or Probiotics on Human Colonic Metabolism: Does It Affect Human Health? Molecular Nutrition \& Food Research, 2011. 55(1): p. 46-57.

[14] Gibson, G.R., From Probiotics to Prebiotics and a Healthy Digestive System. Journal of Food Science, 2004. 69(5): p. M141-M143.

[15] Schrezenmeir, J. and M. de Vrese, Probiotics, Prebiotics, and Synbiotics - Approaching a Definition. The American Journal of Clinical Nutrition, 2001. 73(2): p. 361S-364S.

[16] Tuohy, K.M., et al., Modulation of the Human Gut Microflora Towards Improved Health Using Prebiotics - Assessment of Efficacy. Current Pharmaceutical Design, 2005. 11: p. 75-90.

[17] Rastall, R.A., Functional Oligosaccharides: Application and Manufacture. Annual Review of Food Science and Technology, 2010. 1(1): p. 305-339.

[18] Sabater-Molina, M., et al., Dietary Fructooligosaccharides and Potential Benefits on Health. Journal of Physiology and Biochemistry, 2009. 65(3): p. 315-328.

[19] Torres, D.P.M., et al., Galacto-Oligosaccharides: Production, Properties, Applications, and Significance as Prebiotics. Comprehensive Reviews in Food Science and Food Safety, 2010. 9(5): p. 438-454.

[20] Champ, M. and C. Hoebler, Functional Food for Pregnant, Lactating Women and in Perinatal Nutrition: A Role for Dietary Fibres? Current Opinion in Clinical Nutrition \& Metabolic Care, 2009. 12(6): p. 565-574.

[21] Caselato de Sousa, V.M., E.F. dos Santos, and V.C. Sgarbieri, The Importance of Prebiotics in Functional Foods and Clinical Practice. Food and Nutrition Sciences, 2011. 2: p. 133-144.

[22] Bosscher, D., J. Van Loo, and A. Franck, Inulin and Oligofructose as Prebiotics in the Prevention of Intestinal Infections and Diseases. Nutrition Research Reviews, 2006. 19: p. 216-226.

[23] Panesar, P.S. and S. Kumari, Lactulose: Production, Purification and Potential Applications. Biotechnology Advances, 2011. 29(6): p. 940-948.

[24] Flamm, G., et al., Inulin and Oligofructose as Dietary Fiber: A Review of the Evidence. Critical Reviews in Food Science and Nutrition, 2001. 41(5): p. 353-362. 
[25] Van den Ende, W., D. Peshev, and L. De Gara, Disease Prevention by Natural Antioxidants and Prebiotics Acting as ROS Scavengers in the Gastrointestinal Tract. Trends in Food Science \& Technology, 2011. 22(12): p. 689-697.

[26] Van Loo, J., et al., Functional Food Properties of Non-Digestible Oligosaccharides: A Consensus Report from the ENDO Project (DGXII AIRII-CT94-1095). British Journal of Nutrition, 1999. 81: p. 121-132.

[27] Knol, J., et al., Colon Microflora in Infants Fed Formula with Galacto- and FructoOligosaccharides: More Like Breast-Fed Infants. Journal of Pediatric Gastroenterology and Nutrition, 2005. 40(1): p. 36-42.

[28] Gopal, P.K., P.A. Sullivan, and J.B. Smart, Utilisation of Galacto-Oligosaccharides as Selective Substrates for Growth by Lactic Acid Bacteria Including Bifidobacterium lactis DR10 and Lactobacillus rhamnosus DR20. International Dairy Journal, 2001. 11(1-2): p. 19-25.

[29] Kaplan, H. and R.W. Hutkins, Fermentation of Fructooligosaccharides by Lactic Acid Bacteria and Bifidobacteria. Applied and Environmental Microbiology, 2000. 66(6): p. 2682-2684.

[30] Kaplan, H. and R.W. Hutkins, Metabolism of Fructooligosaccharides by Lactobacillus paracasei 1195. Applied and Environmental Microbiology, 2003. 69(4): p. 2217-2222.

[31] Searle, L.E.J., et al., Purified Galactooligosaccharide, Derived from a Mixture Produced by the Enzymic Activity of Bifidobacterium bifidum, Reduces Salmonella enterica Serovar Typhimurium Adhesion and Invasion In Vitro and In Vivo. Journal of Medical Microbiology, 2010. 59(12): p. 1428-1439.

[32] Shoaf, K., et al., Prebiotic Galactooligosaccharides Reduce Adherence of Enteropathogenic Escherichia coli to Tissue Culture Cells. Infection and Immunity, 2006. 74(12): p. 6920-6928.

[33] Mountzouris, K.C., et al., Modeling of Oligodextran Production in an Ultrafiltration Stirred-Cell Membrane Reactor. Enzyme and Microbial Technology, 1999. 24(1-2): p. 75-85.

[34] Rycroft, C.E., et al., A Comparative In Vitro Evaluation of the Fermentation Properties of Prebiotic Oligosaccharides. Journal of Applied Microbiology, 2001. 91(5): p. 878-887.

[35] Kaneko, T., et al., Effects of Isomaltooligosaccharides with Different Degrees of Polymerization on Human Fecal Bifidobacteria. Bioscience, Biotechnology, and Biochemistry, 1994. 58(12): p. 2288-2290.

[36] Saito, Y., T. Takano, and I. Rowland, Effects of Soybean Oligosaccharides on the Human Gut Microflora in In Vitro Culture. Microbial Ecology in Health and Disease, 1992. 5(2): p. 105-110.

[37] Sharp, R., S. Fishbain, and G.T. Macfarlane, Effect of Short-Chain Carbohydrates on Human Intestinal Bifidobacteria and Escherichia coli In Vitro. Journal of Medical Microbiology, 2001. 50(2): p. 152-160.

[38] Hopkins, M.J., J.H. Cummings, and G.T. Macfarlane, Inter-Species Differences in Maximum Specific Growth Rates and Cell Yields of Bifidobacteria Cultured on Oligosaccharides and Other Simple Carbohydrate Sources. Journal of Applied Microbiology, 1998. 85(2): p. 381-386.

[39] Hopkins, M.J. and G.T. Macfarlane, Changes in Predominant Bacterial Populations in Human Faeces with Age and with Clostridium difficile Infection. Journal of Medical Microbiology, 2002. 51(5): p. 448-454. 
[40] Fuentes-Zaragoza, E., et al., Resistant Starch as Prebiotic: A Review. Starch - Starke, 2011. 63(7): p. 406-415.

[41] Hernandez-Hernandez, O., et al., Characterization of Galactooligosaccharides Derived from Lactulose. Journal of Chromatography A, 2011. 1218(42): p. 7691-7696.

[42] Lesmes, U., et al., Effects of Resistant Starch Type III Polymorphs on Human Colon Microbiota and Short Chain Fatty Acids in Human Gut Models. Journal of Agricultural and Food Chemistry, 2008. 56(13): p. 5415-5421.

[43] Hur, S.J., et al., In Vitro Human Digestion Models for Food Applications. Food Chemistry, 2011. 125(1): p. 1-12.

[44] Kong, F. and R.P. Singh, A Human Gastric Simulator (HGS) to Study Food Digestion in Human Stomach. Journal of Food Science, 2010. 75(9): p. E627-E635.

[45] Macfarlane, G.T. and S. Macfarlane, Models For Intestinal Fermentation: Association between Food Components, Delivery Systems, Bioavailability and Functional Interactions in the Gut. Current Opinion in Biotechnology, 2007. 18(2): p. 156-162.

[46] Benshitrit, R.C., et al., Development of Oral Food-Grade Delivery Systems: Current Knowledge and Future Challenges. Food \& Function, 2012. 3(1): p. 10-21.

[47] Molly, K., et al., Validation of the Simulator of the Human Intestinal Microbial Ecosystem (SHIME) Reactor Using Microorganism-associated Activities. Microbial Ecology in Health and Disease, 1994. 7(4): p. 191-200.

[48] De Boever, P., B. Deplancke, and W. Verstraete, Fermentation by Gut Microbiota Cultured in a Simulator of the Human Intestinal Microbial Ecosystem Is Improved by Supplementing a Soygerm Powder. The Journal of Nutrition, 2000. 130(10): p. 2599-2606.

[49] Gmeiner, M., et al., Influence of a Synbiotic Mixture consisting of Lactobacillus acidophilus 74-2 and a Fructooligosaccharide Preparation on the Microbial Ecology Sustained in a Simulation of the Human Intestinal Microbial Ecosystem (SHIME Reactor). Applied Microbiology and Biotechnology, 2000. 53(2): p. 219-223.

[50] Minekus, M., et al., A Multicompartmental Dynamic Computer-Controlled Model Simulating the Stomach and Small Intestine. Atla Alternatives To Laboratory Animals, 1995. 23(2): p. 197-209.

[51] Macfarlane, G.T., S. Macfarlane, and G.R. Gibson, Validation of a Three-Stage Compound Continuous Culture System for Investigating the Effect of Retention Time on the Ecology and Metabolism of Bacteria in the Human Colon. Microbial Ecology, 1998. 35(2): p. 180-187.

[52] Delzenne, N.M., et al., Targeting Gut Microbiota in Obesity: Effects of Prebiotics and Probiotics. Nature Reviews Endocrinology, 2011. 7(11): p. 639-646.

[53] Chichlowski, M., et al., The Influence of Milk Oligosaccharides on Microbiota of Infants: Opportunities for Formulas. Annual Review of Food Science and Technology, 2011. 2(1): p. 331-351.

[54] Marques, T.M., et al., Programming Infant Gut Microbiota: Influence of Dietary and Environmental Factors. Current Opinion in Biotechnology, 2010. 21(2): p. 149-156.

[55] Morelli, L., Postnatal Development of Intestinal Microflora as Influenced by Infant Nutrition. The Journal of Nutrition, 2008. 138(9): p. 1791S-1795S.

[56] Adlerberth, I. and A.E. Wold, Establishment of the Gut Microbiota in Western Infants. Acta Pædiatrica, 2009. 98(2): p. 229-238. 
[57] Penders, J., et al., Factors Influencing the Composition of the Intestinal Microbiota in Early Infancy. Pediatrics, 2006. 118(2): p. 511-521.

[58] Palmer, C., et al., Development of the Human Infant Intestinal Microbiota. PLoS Biol, 2007. 5(7): p. e177.

[59] Harmsen, H.J.M., et al., Analysis of Intestinal Flora Development in Breast-Fed and Formula-Fed Infants by Using Molecular Identification and Detection Methods Journal of Pediatric Gastroenterology \& Nutrition, 2000. 30(1): p. 61-67.

[60] Rubaltelli, F.F., et al., Intestinal Flora in Breast- and Bottle-Fed Infants. Journal of Pediatric Gastroenterology \& Nutrition, 1998. 26(3): p. 186-191.

[61] Moro, G., et al., Dosage-Related Bifidogenic Effects of Galacto- and Fructooligosaccharides in Formula-Fed Term Infants. Journal of Pediatric Gastroenterology and Nutrition, 2002. 34(3): p. 291-295.

[62] Moro, G.E., et al., Effects of a New Mixture of Prebiotics on Faecal Flora and Stools in Term Infants. Acta Pædiatrica, 2003. 92: p. 77-79.

[63] Fanaro, S., et al., Galacto-Oligosaccharides and Long-Chain Fructo-Oligosaccharides as Prebiotics in Infant Formulas: A Review. Acta Paediatrca Supplement, 2005. 94(449): p. 22-26.

[64] Boehm, G., et al., Supplementation of a Bovine Milk Formula with an Oligosaccharide Mixture Increases Counts of Faecal Bifidobacteria in Preterm Infants. Archives of Disease in Childhood - Fetal and Neonatal Edition, 2002. 86(3): p. F178-F181.

[65] Haarman, M. and J. Knol, Quantitative Real-Time PCR Assays To Identify and Quantify Fecal Bifidobacterium Species in Infants Receiving a Prebiotic Infant Formula. Applied and Environmental Microbiology, 2005. 71(5): p. 2318-2324.

[66] Knol, J., et al., Increase of Faecal Bifidobacteria due to Dietary Oligosaccharides Induces a Reduction of Clinically Relevant Pathogen Germs in the Faeces of Formula-Fed Preterm Infants. Acta Paediatrca Supplement, 2005. 94(449): p. 31-33.

[67] Bouhnik, Y., et al., Administration of Transgalacto-Oligosaccharides Increases Fecal Bifidobacteria and Modifies Colonic Fermentation Metabolism in Healthy Humans. The Journal of Nutrition, 1997. 127(3): p. 444-448.

[68] Salminen, S. and E. Salminen, Lactulose, Lactic Acid Bacteria, Intestinal Microecology and Mucosal Protection. Scandinavian Journal of Gastroenterolgy Supplement, 1997. 222: p. 45-48.

[69] Den Hond, E., B. Geypens, and Y. Ghoos, Effect of High Performance Chicory Inulin on Constipation. Nutrition Research, 2000. 20(5): p. 731-736.

[70] Biagi, E., et al., Ageing of the Human Metaorganism: The Microbial Counterpart. AGE, 2012. 34(1): p. 247-267.

[71] Tiihonen, K., A.C. Ouwehand, and N. Rautonen, Human Intestinal Microbiota and Healthy Ageing. Ageing Research Reviews, 2010. 9(2): p. 107-116.

[72] Cohen, J.E., Human Population: The Next Half Century. Science, 2003. 302(5648): p. 1172-1175.

[73] Christensen, K., et al., Ageing Populations: The Challenges Ahead. The Lancet, 2009. 374(9696): p. 1196-1208.

[74] Woodmansey, E.J., Intestinal Bacteria and Ageing. Journal of Applied Microbiology, 2007. 102(5): p. 1178-1186. 
[75] Guigoz, Y., et al., Effects of Oligosaccharide on the Faecal Flora and Non-Specific Immune System in Elderly People. Nutrition research, 2002. 22(1): p. 13-25.

[76] Bartosch, S., et al., Microbiological Effects of Consuming a Synbiotic Containing Bifidobacterium bifidum, Bifidobacterium lactis, and Oligofructose in Elderly Persons, Determined by Real-Time Polymerase Chain Reaction and Counting of Viable Bacteria. Clinical Infectious Diseases, 2005. 40(1): p. 28-37.

[77] Bouhnik, Y., et al., Four-Week Short Chain Fructo-Oligosaccharides Ingestion Leads to Increasing Fecal Bifidobacteria and Cholesterol Excretion in Healthy Elderly Volunteers. Nutrition Journal, 2007. 6(1): p. 42.

[78] Vulevic, J., et al., Modulation of the Fecal Microflora Profile and Immune Function by a Novel Trans-Galactooligosaccharide Mixture (B-GOS) in Healthy Elderly Volunteers. The American Journal of Clinical Nutrition, 2008. 88(5): p. 1438-1446.

[79] Schiffrin, E.J., et al., Systemic Inflammatory Markers in Older Persons: The Effect of Oral Nutritional Supplementation with Prebiotics. Journal of Nutrition Health \& Aging, 2007. 11(6): p. 475-479.

[80] Fava, F., et al., The Gut Microbiota and Lipid Metabolism: Implications for Human Health and Coronary Heart Disease. Current Medicinal Chemistry, 2006. 13(25): p. 3005-3021.

[81] Guarner, F. and J.-R. Malagelada, Gut Flora in Health and Disease. The Lancet, 2003. 361(9356): p. 512-519.

[82] Saavedra, J.M. and A. Tschernia, Human Studies with Probiotics and Prebiotics: Clinical Implications. British Journal of Nutrition, 2002. 87: p. S241-S246.

[83] Louis, P. and H.J. Flint, Diversity, Metabolism and Microbial Ecology of ButyrateProducing Bacteria from the Human Large Intestine. FEMS Microbiology Letters, 2009. 294(1): p. 1-8.

[84] McGarr, S.E., J.M. Ridlon, and P.B. Hylemon, Diet, Anaerobic Bacterial Metabolism, and Colon Cancer: A Review of the Literature. Journal of Clinical Gastroenterology, 2005. 39(2): p. 98-109.

[85] Langlands, S.J., et al., Prebiotic Carbohydrates Modify the Mucosa Associated Microflora of the Human Large Bowel. Gut, 2004. 53(11): p. 1610-1616.

[86] Hotamisligil, G.S., Inflammation and Endoplasmic Reticulum Stress in Obesity and Diabetes. International Journal of Obesity, 2008. 32(S7): p. S52-S54.

[87] Shoelson, S.E. and A.B. Goldfine, Getting Away from Glucose: Fanning the Flames of Obesity-Induced Inflammation. Nature Medicine, 2009. 15(4): p. 373-374.

[88] Cani, P.D. and N.M. Delzenne, Interplay Between Obesity and Associated Metabolic Disorders: New Insights into the Gut Microbiota. Current Opinion in Pharmacology, 2009. 9(6): p. 737-743.

[89] Cani, P.D., et al., Improvement of Glucose Tolerance and Hepatic Insulin Sensitivity by Oligofructose Requires a Functional Glucagon-Like Peptide 1 Receptor. Diabetes, 2006. 55(5): p. 1484-1490.

[90] Cani, P.D., et al., Oligofructose Promotes Satiety in Rats Fed a High-Fat Diet: Involvement of Glucagon-like Peptide-1. Obesity Research, 2005. 13(6): p. 1000-1007.

[91] Cani, P.D., et al., Changes in Gut Microbiota Control Inflammation in Obese Mice through a Mechanism involving GLP-2-Driven Improvement of Gut Permeability. Gut, 2009. 58: p. 1091-1128. 
[92] Daubioul, C., et al., Dietary Fructans, but Not Cellulose, Decrease Triglyceride Accumulation in the Liver of Obese Zucker fa/fa Rats. The Journal of Nutrition, 2002. 132(5): p. 967-973.

[93] Daubioul, C.A., et al., Dietary Oligofructose Lessens Hepatic Steatosis, but Does Not Prevent Hypertriglyceridemia in Obese Zucker Rats. The Journal of Nutrition, 2000. 130(5): p. 1314-1319.

[94] Delmee, E., et al., Relation between Colonic Proglucagon Expression and Metabolic Response to Oligofructose in High Fat Diet-Fed Mice. Life Sciences, 2006. 79(10): p. 1007-1013.

[95] Roberfroid, M., et al., Prebiotic Effects: Metabolic and Health Benefits. British Journal of Nutrition, 2010. 104: p. S1-S63

[96] Abrams, S.A., et al., Effect of Prebiotic Supplementation and Calcium Intake on Body Mass Index. The Journal of Pediatrics, 2007. 151(3): p. 293-298.

[97] Maurer, A.D., et al., Changes in Satiety Hormones and Expression of Genes Involved in Glucose and Lipid Metabolism in Rats Weaned Onto Diets High in Fibre or Protein Reflect Susceptibility to Increased Fat Mass in Adulthood. The Journal of Physiology, 2009. 587(3): p. 679-691.

[98] Maurer, A., et al., Consumption of Diets High in Prebiotic Fiber or Protein During Growth Influences the Response to a High Fat and Sucrose Diet in Adulthood in Rats. Nutrition \& Metabolism, 2010. 7(1): p. 77.

[99] Kalliomaki, M., et al., Early Differences in Fecal Microbiota Composition in Children May Predict Overweight. The American Journal of Clinical Nutrition, 2008. 87(3): p. 534-538.

[100] Cani, P.D., C. Dewever, and N.M. Delzenne, Inulin-Type Fructans Modulate Gastrointestinal Peptides Involved in Appetite Regulation (Glucagon-Like Peptide-1 and Ghrelin) in Rats. British Journal of Nutrition, 2004. 92(3): p. 521-526.

[101] Wu, X., et al., Molecular Characterisation of the Faecal Microbiota in Patients with Type II Diabetes. Current Microbiology, 2010. 61(1): p. 69-78.

[102] Delzenne, N.M. and C.M. Williams, Prebiotics and Lipid Metabolism. Cuurent Opinion in Lipidology, 2002. 13(1): p. 61-67.

[103] Cani, P.D., et al., Metabolic Endotoxemia Initiates Obesity and Insulin Resistance. Diabetes, 2007. 56(7): p. 1761-1772.

[104] Cani, P.D. and N.M. Delzenne, The Gut Microbiome as Therapeutic Target. Pharmacology \& Therapeutics, 2011. 130(2): p. 202-212.

[105] Wong, J.M.W., et al., Colonic Health: Fermentation and Short Chain Fatty Acids. Journal of Clinical Gastroenterology, 2006. 40(3): p. 235-243.

[106] Gao, Z., et al., Butyrate Improves Insulin Sensitivity and Increases Energy Expenditure in Mice. Diabetes, 2009. 58(7): p. 1509-1517.

[107] Cani, P.D., et al., Oligofructose Promotes Satiety in Healthy Human: A Pilot Study. European Journal of Clinical Nutrition, 2005. 60(5): p. 567-572.

[108] Cani, P.D., et al., Gut Microbiota Fermentation of Prebiotics Increases Satietogenic and Incretin Gut Peptide Production with Consequences for Appetite Sensation and Glucose Response After a Meal. The American Journal of Clinical Nutrition, 2009. 90(5): p. 1236-1243. 
[109] Whelan, K., et al., Appetite During Consumption of Enteral Formula as a Sole Source of Nutrition: The Effect of Supplementing Pea-Fibre and Fructo-Oligosaccharides. British Journal of Nutrition, 2006. 96(2): p. 350-356.

[110] Chaudhri, O.B., B.C.T. Field, and S.R. Bloom, Gastrointestinal Satiety Signals. International Journal of Obesity, 2008. 32(S7): p. S28-S31.

[111] Cowley, M.A., et al., The Distribution and Mechanism of Action of Ghrelin in the CNS Demonstrates a Novel Hypothalamic Circuit Regulating Energy Homeostasis. Neuron, 2003. 37(4): p. 649-661.

[112] Druce, M.R., C.J. Small, and S.R. Bloom, Minireview: Gut Peptides Regulating Satiety. Endocrinology, 2004. 145(6): p. 2660-2665.

[113] Wynne, K., et al., Appetite Control. Journal of Endocrinology, 2005. 184(2): p. 291-318.

[114] Freeland, K.R., C. Wilson, and T.M. Wolever, Adaptation of Colonic Fermentation and Glucagon-Like Peptide-1 Secretion with Increased Wheat Fibre Intake for 1 Year in Hyperinsulinaemic Human Subjects. British Journal of Nutrition, 2010. 103(1): p. 82-90.

[115] Freeland, K.R. and T.M. Wolever, Acute Effects of Intravenous and Rectal Acetate on Glucagon-Like Peptide-1, Peptide YY, Ghrelin, Adiponectin and Tumour Necrosis Factor-Alpha. British Journal of Nutrition, 2010. 103(3): p. 460-466.

[116] Piche, T., et al., Colonic Fermentation Influences Lower Esophageal Sphincter Function in Gastroesophageal Reflux Disease. Gastroenterology, 2003. 124(4): p. 894-902.

[117] Parnell, J.A. and R.A. Reimer, Weight Loss During Oligofructose Supplementation is Associated with Decreased Ghrelin and Increased Peptide YY in Overweight and Obese Adults. The American Journal of Clinical Nutrition, 2009. 89(6): p. 1751-1759.

[118] Tarini, J. and T.M.S. Wolever, The Fermentable Fibre Inulin Increases Postprandial Serum Short-Chain Fatty Acids and Reduces Free-Fatty Acids and Ghrelin in Healthy Subjects. Applied Physiology, Nutrition, and Metabolism, 2010. 35(1): p. 9-16.

[119] Woodmansey, E.J., et al., Comparison of Compositions and Metabolic Activities of Fecal Microbiotas in Young Adults and in Antibiotic-Treated and Non-AntibioticTreated Elderly Subjects. Applied and Environmental Microbiology, 2004. 70(10): p. 6113-6122.

[120] Mueller, S., et al., Differences in Fecal Microbiota in Different European Study Populations in Relation to Age, Gender, and Country: A Cross-Sectional Study. Applied and Environmental Microbiology, 2006. 72(2): p. 1027-1033.

[121] Claesson, M.J., et al., Comparative Analysis of Pyrosequencing and a Phylogenetic Microarray for Exploring Microbial Community Structures in the Human Distal Intestine. PLoS ONE, 2009. 4(8): p. e6669.

[122] Mariat, D., et al., The Firmicutes/Bacteroidetes ratio of the human microbiota changes with age. BMC Microbiology, 2009. 9(1): p. 123.

[123] Reinhardt, C., C.S. Reigstad, and F. Backhed, Intestinal Microbiota During Infancy and Its Implications for Obesity. Journal of Pediatric Gastroenterology and Nutrition, 2009. 48(3): p. 249-256

[124] Biasucci, G., et al., Cesarean Delivery May Affect the Early Biodiversity of Intestinal Bacteria. The Journal of Nutrition, 2008. 138(9): p. 1796S-1800S. 